type $\mathrm{CO}$ but a whole range of small and big molecules -, and catalytic reactions. The whole spectrum of surface diagnostic tools seems to be used to probe primarily the end products of the reactions or possible intermediates. There were also reports on processes that give information about the early stages of reactions, for instance surface chemiluminescence.

Invited talks and contributed papers on catalysis, the insulator-semiconductor interface, metallurgy, friction, and lubrication gave an indication of the central role played by Surface Science.

When looking at the abstracts and posters or listening to the talks, the symptoms of a creeping disease of Surface Science are seen: LEED, MEED, RHEED, SIMS, AES, XPS, HRELS, UP, TPTD, ESCA, TEM, ESDIAD, ISS, SXAPS, EELS, ARUPS, SRPS, SPLEED, FEM, TD, ESD, DTA, ISS, SAM ,FEED, MBE, RED, SEM, SCANIIR, BLE, MEIS, SES, MEM, LID,
LEIS, RESLEET, INS, ..... Some people claim that these symbols stand for useful experimental techniques, and others say things like " ... the EDC from UPS under UHV relates to the DOS." The question is whether we should and could stop the exponential growth in these "four letter" words.

Statistics about Surface Science in Europe ') gathered from this and recent conferences and the journal Surface Science, indicate amongst other things - an under-representation from eastern Europe, with however a positive gradient, and a decrease in contributions from industrial laboratories. This last should be seen as a warning for future annual meetings of the ECOSS series, especially as the coordination aims have been expressed as : "... to serve the needs and aspirations of European research workers in the field of Surface Science and to promote their communication, $\ldots$ and to ensure a high scientific standard, the interdisciplinary nature of the field and the inclusion of new developments in both fundamental and applied areas "1).

The next opportunities to work towards these aims will be at

- ECOSS II in Cambridge, England, 26-29 March, 1979

- ECOSS III in Cannes, France, 2127 September, 1980

- ECOSS IV in Germany, 1981.

The "supermarkets with posters" ") of ECOSS I might then be partly abandoned, whilst still avoiding parallel sessions. Surface Science is in too rapid a state of development to allow divisions into fixed branches. Crossfertilization is a MUST in this active field.

\section{Footnotes :}

1. Welcome remarks by G.A. Bootsma, President of the Nederlandse Vacuumvereiniging.

2. ECOSS I, Book of Abstracts, in Nederlands Tijdschrift voor Vacuum Techniek, 16e, Jaargang Nr. 2/3/4 (1978).

B. Lundqvist

(University of Aarhus)

\title{
10th European Conference on Atomic Spectroscopy
}

As usual, the annual meeting of the "European Group for Atomic Spectroscopy (EGAS)" took place in the second week of July. Attended by 264 spectroscopists from 19 countries, both West and East, splendidly organized at the Garching Research Centre by $\mathrm{H}$. Walther and his colleagues from the Physics Department of the University of Munich and the Max-PlanckInstitut for Plasmaphysics at Garching, this was a fitting occasion for the 10th anniversary. EGAS, a Section of the Atomic Physics Division of EPS, in its development has, from the beginning, kept a style of its own. When founded at Caen (Normandy) its main aims were formulated as being to :

(i) bring together regularly European atomic spectroscopists in order to foster the exchange of results and ideas and, different from many other societies and groups.

(ii) give especially the younger colleagues a chance - a theme constantly repeated.

One main trend of present scientific work is that the laser seems no longer to be mainly an object of research per se, but rather has become a research tool for obtaining results in atomic, nuclear and also fundamental physics. Here W. Demtröder reviewed the latest state of high resolution or even sub-Doppler spectroscopy and the more sensitive detection techniques as, e.g., saturation interference spectroscopy within a Jamin-Interferometer.

For one of these newer methods "Fast Ion Beam Laser Spectroscopy," where the Doppler width is reduced by " acceleration cooling," the basic principles, essential requirements and significance were dealt with in detail by M.L. Gaillard and in a number of contributed papers. For the future, important results may be expected for high resolution spectroscopy of atoms and molecules, for collisions in excited states, and for hyperfinestructure (hfs) research in "exotic" atoms.

The importance of the laser as a tool became further manifest by the fact that many - also more conventional - applications of lasers were reported in a large number of sessions and papers. They were concerned, e.g., with hfs and isotope shift (IS) investigations, lifetime measurements, the search for the first optical transition in francium or the determination of the fine structure (FS) separation of high Rydberg states by quantum beats (applying an electric field for the detection).
For such highly excited atoms in electric fields S. Feneuille reviewed the theoretical treatment and experimental facts much of which can be understood only recently.

A further new way of experimenting was also sketched : it connects atomic and nuclear physics beyond the traditional coupling through shell-nucleus interaction (hfs and IS). As demonstrated by E.W. Otten and his group impressive results on the nucleus (e.g. $\delta<r^{2}>$ ) can be obtained by combining optical pumping with nuclear research techniques ( $\beta$-decay). Thus systematic hfs and IS investigations were possible of several shortlived isotopes with the laser in line with an ion beam, as in ISOLDE at CERN.

In future, laser applications may also be decisive for the fundamental question of parity violation in atomic physics. Mme M.A. Bouchiat, (one of the originators of research into parity non-conserving effects in atomic physics) reviewed the present confusing situation. Of three experiments to measure the optical rotation of $\mathrm{Bi}$ atoms (Oxford, Seattle, Novosibirsk) only in the Soviet experiment were the rotations said to be consistent with theory. On the other hand, in a 
recent experiment with scattered electrons at the Stanford Linear Accelerator, an asymmetry in agreement with theory was found. New experiments now underway could solve this problem within a few months.

It became very clear, that the more traditional spectroscopic and interference spectroscopic methods have, of late, been stimulated by new techniques and problems. Thus research in astrophysics has recently raised the need for more "spectra of highly stripped ions" which can now be generated in laser produced plasmas as reported by U. Feldman. W.R.S. Garton's research work in which, by means of short wavelength synchrotron radiation, spectra of inner and doubly excited electrons are obtained and investigated, also shows clearly the importance of true spectroscopy for progress in atomic physics. This is just one reason for extending classical level classification work and completing the spectra so far only partly known. This work is obviously profiting from more and more powerful computer programmes.

Generally it was felt and emphasized in round table discussions that there again exists a definite and strong challenge to classical optical spectroscopy, to obtain systematic data for nearly all transitions, levels, terms and spectra. These data, even if of only moderate accuracy, are sometimes the most valuable to theoreticians. On the other hand, some modern methods are now so refined that they yield data of once quite incredible accuracy $\left(10^{-10}\right)$, so that, even 16-pole nuclear moments could be measured and reported.

Roland Winkler (Berlin Technical University)

\section{Physics of the Actinides}

The Third International Conference on the Electronic Structure of the Actinides, AN 78, which took place on the University campus of Grenoble from 30 August to 1 September, belongs to a series which started in Argonne in 1974, followed by Wroclaw in 1976. The next conference will most likely be held near Berkeley in 1981. Organized jointly by the Centre d'Etudes Nucléaires de Grenoble of the CEA and the European Institute of Transuranium Elements at Karlsruhe, AN 78 gathered together 130 participants from 14 countries (mainly European).

The scientific programme was divided into six sessions, each corresponding to one half day and consisting of two invited talks and poster communications. The themes of the sessions indicate the ground covered:

1. Physical properties of metallic magnetic compounds, 5f-band approach:

2. Physical properties of metallic magnetic compounds, 5f-localized approach;

3. Sample preparation and characterization for physical measurements;

4. Physical properties of metals and non-magnetic alloys;

5. Physical properties of ions, ionic and semi-conducting compounds;

6. Correlations between electronic structure, thermodynamics and crystal structure.

Three panel discussions were also

held upon the following topics:

Aspects of the $5 f$ electrons (de) localization;

Availability and sample preparation; limits of solid state studies on actinides

"NACl" structure actinide compounds.

In this conference centred on the condensed matter physics of the ac- tinides, great importance was attached to the chemistry. This is due to a number of factors. First, because the transuranium elements do not exist in nature and have to be prepared through nuclear reactions (mainly in neutron reactors), weighable amounts of actinides are available only up to einsteinium, which sets the limit for solid state studies. Only $10^{10}$ atoms of the next actinide, fermium, are available. Second, the preparation and characterization of actinide compounds necessitate, apart from special equipment found only in highly radioactive laboratories, the use of techniques adapted to the high cost and scarcity of the actinide materials which increases progressively as we go to the right of the actinide series: $\mathrm{kg}$ quantities of ${ }^{244} \mathrm{Cm}, \mathrm{g}$ quantities of ${ }^{252} \mathrm{Cf}$; mg quantities of ${ }^{253} \mathrm{Es},{ }^{249} \mathrm{Cf}$, ${ }^{248} \mathrm{Cm}$, total in the world. Third, it appears that for many experimental techniques (magnetization, transport, optical, X ray, neutron), single crystals are not just very useful but often necessary. These are precisely the techniques from amongst which we hope to see major advances in actinide physics.

From an experimental viewpoint indeed, the availability of sufficiently large uranium single crystals has been the stimulus for many of the detailed studies presented. This is especially true for the "NACl" type compounds, where neutron studies have emerged as a major technique in the actinide field. Both neutron and magnetic measurements revealed the importance of anisotropy effects, and gave valuable insight into the valency and spatial character of $5 f$ electrons (localized versus itinerant).

From the theoretical viewpoint, new results have been presented on the electronic structure of actinides arriv- ed at mainly through band structure calculations; the complex $\alpha$-phase of uranium has been attacked successfully. Attempts have been made also to utilize more physically transparent methods, one result being the calculation of bulk quantities such as the atomic volume of actinide pure metals. The bonding contribution from $5 f$ electrons was shown to be prominent in the beginning of the actinide series, in agreement with their complex crystallographic structure.

For the future, two conclusions can be drawn from the Conference:

- The extension of single crystal preparation for transuranium compounds is a major need for further progress to be made; AN 78 remained essentially a "Uranium" conference.

- Apart from a foreseeable development of neutron experiments, optical experiments (especially photoemission techniques) should develop and give some contribution to the still open subject of $5 f$ electrons (de) localization.

\section{Belakhovsky}

(Centre d'Etudes Nucléaires, Grenoble)

\section{UNIVERSITY OF GENEVA}

A post-doctoral research appointment is available at the Laboratory for Applied Chemistry for a solid state physicist or solid state chemist interested in joining an experimental programme (Swiss National Science Foundation) on the synthesis and the properties of ferroelectric and related magnetic materials. A three years' programme is foreseen. Renewal of this appointment is possible from year to year.

Applicants should submit résumé publication list and names of two referees to: Prof. H. Schmid, Applied Chemistry Laboratory, University of Geneva, 30, Quai ErnestAnsermet, CH-1211 Geneva 4. 